\title{
FIXED-POINT SETS OF AUTOHOMEOMORPHISMS OF COMPACT $F$-SPACES
}

\author{
K. P. HART AND J. VERMEER \\ (Communicated by Franklin D. Tall)
}

\begin{abstract}
We investigate fixed-point sets of autohomeomorphisms of compact $F$-spaces. If the space in question is finite dimensional (in the sense of covering dimension), then the fixed-point set is a $P$-set; on the other hand there is an infinite-dimensional compact $F$-space with an involution whose fixed-point set is not a $P$-set.

In addition we show that under $\mathrm{CH}$ a closed subset of $\omega^{*}$ is a $P$-set iff it is the fixed-point set of an autohomeomorphism.
\end{abstract}

\section{INTRODUCTION}

In this note we investigate the fixed-point sets of autohomeomorphisms of compact $F$-spaces. In Vermeer $[6,7]$ the second author studied fixed-point sets of continuous self-maps of extremally and basically disconnected spaces. It was proved that whenever $X$ is a compact $\kappa$-basically disconnected space (i.e., the Stone space of a $\kappa$-complete Boolean algebra) and $\phi: X \rightarrow X$ is injective and continuous, the fixed-point set of $\phi$ is a $P_{\kappa}$-set of $X$. In particular for a basically disconnected (i.e., $\omega_{1}$-basically disconnected) space the fixed-point set of a self-embedding is always a $P$-set.

The methods used to obtain the above-mentioned result do not readily generalize to the natural extension of the class of basically disconnected spaces: the class of $F$-spaces. The point is that these methods relied heavily on the fact that a countable increasing union of clopen sets in a basically disconnected space has a clopen closure and this last property hardly ever holds nontrivially in general $F$-spaces.

Here we use results about fixed-point free extensions of fixed-point free maps to obtain the result that the fixed-point set of an autohomeomorphism of a finitedimensional compact $F$-space is a $P$-set of that space. This seems to be new, even for the space $\omega^{*}$.

If we assume the Continuum Hypothesis, then we can even show that a closed subset of $\omega^{*}$ is a $P$-set iff it is the fixed-point set of an autohomeomorphism

Received by the editors April 19, 1993.

1991 Mathematics Subject Classification. Primary 54G05; Secondary 54H25.

Key words and phrases. F-space, fixed point, Čech-Stone compactification. 
(even an involution) of $\omega^{*}$. This gives an external characterization of the $P$ sets in $\omega^{*}$ and is a partial answer to Problem 218 of Hart and van Mill [4]. We finish the paper with an example of an infinite-dimensional compact $F$-space and an involution on it whose fixed-point set is not a $P$-set.

\section{Preliminaries}

By convention all spaces under consideration are completely regular. We call-as usual-a space $X$ an $F$-space if every cozero set in it is $C^{*}$-embedded, i.e., if $M$ is a cozero set of $X$ and $f: M \rightarrow \mathbb{R}$ is a bounded continuous function, then $f$ can be extended to a bounded continuous function from $X$ to $\mathbb{R}$. For compact spaces this takes the following convenient form: A compact space $X$ is an $F$-space iff for every $F_{\sigma}$-subset $F$ of $X$ the equality cl $F=\beta F$ holds. A rich supply of compact $F$-spaces can be gotten from the well-known fact that $\beta X \backslash X$ is an $F$-space whenever $X$ is $\sigma$-compact and locally compact.

We also need the characterization of $\omega^{*}$ given by Parovičenko in [5]. This characterization is valid under the assumption of the Continuum Hypothesis $(\mathrm{CH})$.

Theorem 1.1 (CH). A compact space $X$ is homeomorphic to $\omega^{*}$ if and only if it is a compact, zero-dimensional $F$-space of weight $\mathfrak{c}$ without isolated points in which nonempty $G_{\delta}$-sets have nonempty interiors.

This theorem is particularly useful when one works with $P$-sets in $\omega^{*}$; we recall that a subset of a space is a $P$-set if every $G_{\delta}$-set containing it is a neighbourhood of it or, equivalently, a set $A$ is a $P$-set if for every $F_{\sigma}$-set $F$ disjoint from it one has $A \cap \mathrm{cl} F=\varnothing$.

For example, in the proof of Lemma 1.3 below we use the fact that $\omega^{*} \backslash$ Int $A$ is homeomorphic to $\omega^{*}$ whenever $A$ is a $P$-set of $\omega^{*}$. A second application occurs in the proof of Theorem 2.2.

From van Douwen and van Mill [2] we quote the following theorem, the homeomorphism extension theorem for nowhere dense $P$-sets.

Theorem 1.2 $(\mathrm{CH})$. Let $A$ and $B$ be nowhere dense $P$-sets of $\omega^{*}$ and $h: A \rightarrow$ $B$ a homeomorphism. Then there is an autohomeomorphism $\tilde{h}$ of $\omega^{*}$ that extends $h$.

We shall need the following mild extension of this theorem.

Lemma $1.3(\mathrm{CH})$. Let $A$ and $B$ be proper P-subsets of $\omega^{*}$, and let $h: A \rightarrow B$ be a homeomorphism that maps the interior of $A$ onto the interior of $B$. Then there is an autohomeomorphism $\tilde{h}$ of $\omega^{*}$ that extends $h$.

Proof. Consider $\omega^{*} \backslash$ Int $A$ and $\omega^{*} \backslash \operatorname{Int} B$. As noted above both spaces are homeomorphic to $\omega^{*}$ because $A$ and $B$ are $P$-sets.

The homeomorphism extension theorem for nowhere dense $P$-sets now gives us an extension $h^{\prime}: \omega^{*} \backslash$ Int $A \rightarrow \omega^{*} \backslash$ Int $B$ of the restriction $h\lceil\operatorname{Fr} A$. To finish we let $\tilde{h}=h \cup h^{\prime}$.

The final result that we need is from van Douwen [1]. We use the term 'finite-dimensional' in the sense of the covering dimension dim. 
Theorem 1.4. Let $X$ be a finite-dimensional paracompact space and $f: X \rightarrow X$ a closed self-map for which there is a natural number $k$ such that $\left|f^{-1}(x)\right| \leq k$ for all $x \in X$. Then $f$ has a fixed point if and only if $\beta f$ has a fixed point.

\section{Finite-Dimensional SPACES}

We get our first result by a judicious application of van Douwen's theorem.

Theorem 2.1. Let $X$ be a compact finite-dimensional $F$-space and $\phi: X \rightarrow X$ a continuous and injective map. The fixed-point set $F$ of $\phi$ is a $P$-set of $X$.

Proof. Let $K$ be an $F_{\sigma}$-subset of $X$ that is disjoint from $F$. We must show that $\operatorname{cl} K$ is disjoint from $F$. To this end we take the set $L=\bigcup_{k \in \mathbb{Z}} \phi^{k}[K]$. Observe that $L$ is also an $F_{\sigma}$-set that is disjoint from $F$; that $L$ is an $F_{\sigma}$-set is clear. To see that $L$ contains no fixed points of $\phi$ combine the facts that $K$ contains none and that $\phi$ is injective. It is also clear that $\phi[L] \subseteq L$. Finally we observe that $\phi\left\lceil L\right.$ is closed: use the fact that $\phi^{-1}[L]=L$.

Now, because $X$ is an $F$-space, we have $\operatorname{cl} L=\beta L$. Then van Douwen's theorem implies that $\operatorname{cl} L$ contains no fixed points of $\phi$ either. It follows that cl $L \cap F=\varnothing$, so certainly $\operatorname{cl} K \cap F=\varnothing$.

For the space $\omega^{*}$ we can reverse the implication, provided we assume $\mathrm{CH}$.

Theorem 2.2 $(\mathrm{CH})$. A closed subset $A$ of $\omega^{*}$ is a P-set iff it is the fixed-point set of some autohomeomorphism of $\omega^{*}$.

Proof. Let $A$ be a $P$-set of $\omega^{*}$. We shall find an autohomeomorphism $\phi$ of $\omega^{*}$ of which $A$ is the fixed-point set; indeed, $\phi$ will be an involution, i.e., $\phi^{2}$ is the identity.

Consider $\omega^{*} \times 2$ and identify, for every $x \in A$, the points $\langle x, 0\rangle$ and $\langle x, 1\rangle$ (we glue the two copies of $\omega^{*}$ together along the copies of $A$ ). Because $A$ is a $P$-set, the resulting quotient space $Q$ is homeomorphic to $\omega^{*}$ : it satisfies the conditions from Parovičenko's theorem.

Define an autohomeomorphism $\psi$ of $Q$ by sending $\langle x, i\rangle$ to $\langle x, 1-i\rangle$ for every $x$. Clearly $\psi^{2}$ is the identity and the copy $A_{Q}$ of $A$ in $Q$ is the fixed-point set of $\psi$.

It remains to turn $\psi$ into an autohomeomorphism of $\omega^{*}$ whose fixed-point set is $A$ itself.

The identity Id : $A_{Q} \rightarrow A$ is a homeomorphism that maps the interior of $A_{Q}$ onto the interior of $A$ and so by Lemma 1.3 it may be extended to a homeomorphism $h: Q \rightarrow \omega^{*}$.

In the end we take $\phi=h \circ \psi \circ h^{-1}$ of course.

\section{INFINITE-DIMENSIONAL SPACES}

In this section we give an example of compact infinite-dimensional $F$-space $X$ and an autohomeomorphism $\phi$ of $X$ whose fixed-point set is not a $P$-set. Again $\phi$ can be taken to be an involution.

Our starting point is the following example, considered by van Douwen in [1]. Let $\mathbb{S}=\bigoplus_{n} S^{n}$, where $S^{n}$ is the standard $n$-sphere. Next let $\phi: \mathbb{S} \rightarrow \mathbb{S}$ be the sum of the antipodal mappings. Now $\phi$ has no fixed points, yet $\beta \phi$ does have fixed points; this can be seen as follows: if $\beta \phi$ would have no fixed points, then there would be a finite closed cover $\left\{F_{1}, \ldots, F_{n}\right\}$ of $\beta \mathbb{S}$ such 
that $\beta \phi\left[F_{i}\right] \cap F_{i}=\varnothing$ for all $i$. However, the Lusternik-Schnirelman-Borsuk Theorem (Dugundji and Granas [3, Theorem 4.4]) implies that $\phi\left[F_{i}\right] \cap F_{i} \cap S^{n} \neq$ $\varnothing$ for some $i$.

To begin we take for every $n$ the closed $n$-ball $B^{n}$. Remove the origin and call the result $X_{n}$. The antipodal map $e_{n}$ on $X_{n}$ has no fixed points and, as $\operatorname{dim} X_{n}=n$, neither do $\beta e_{n}$ and $e_{n}^{*}=\beta e_{n} \mid X_{n}^{*}$ (apply Theorem 1.4). Also note that $X_{n}^{*}$ is an $F$-space.

Write $X=\bigoplus_{n} \beta X_{n}$ and $e=\bigoplus_{n} \beta e_{n}$. The map $e$ has no fixed points but $\beta e$ has many of them: for any sequence $\left\langle S_{n}\right\rangle_{n}$ of spheres centered at the origins of the $B^{n}$ we get fixed points of $\beta e$ in the closure of $\bigoplus_{n} S_{n}$.

Now take any neighbourhood of $\left(\bigoplus_{n} X_{n}^{*}\right)^{*}$ in $\beta X$; it contains a tail of a sequence of spheres as in the preceding paragraph and hence a fixed point of $\beta e$. But then $\left(\bigoplus_{n} X_{n}^{*}\right)^{*}$ contains fixed points of $\beta e$ as well.

Our example is the closure of $\bigoplus_{n} X_{n}^{*}$ in $\beta X$, and the map $\phi$ is the restriction of $\beta e$. It is clearly an $F$-space, and the (nonempty) fixed-point set of $\phi$ is contained in the nowhere dense $G_{\delta}$-set $\left(\bigoplus_{n} X_{n}^{*}\right)^{*}$.

\section{REFERENCES}

1. E. K. van Douwen, $\beta X$ and fixed-point free maps, Topology Appl. 51 (1993), 191-195.

2. E. K. van Douwen and J. van Mill, The homeomorphism extension theorem for $\beta \omega \backslash \omega$, Ann. New York Acad. Sci. 704 (1993), 345-350

3. J. Dugundji and A. Granas Fixed point theory, Vol. I, Monograf. Mat., PWN, Warszawa, 1982.

4. K. P. Hart and J. van Mill, Open problems on $\beta \omega$, Open Problems in Topology (J. van Mill and G. M. Reed, eds.), North-Holland, Amsterdam, 1990, pp. 97-125.

5. I. I. Parovičenko, $A$ universal bicompact of weight $\aleph$, Soviet Math. Dokl. 4 (1963), 592-592.

6. J. Vermeer, Frolik's theorem for basically disconnected spaces, Acta Univ. Carolin.-Math. Phys. 34 (1993), 135-142.

7. _ Fixed-point sets of continuous functions of extremally disconnected spaces, Trans. Amer. Math. Soc. (to appear).

Faculty of Technical Mathematics and Informatics, TU Delft, Postbus 5031, 2600 Ga DELFT, THE NeTHERLANDS

E-mail address: wiawkph@dutrun2.tudelft.nl

E-mail address: J.Vermeer@twi.tudelft.nl 